\title{
5 Research Square

\section{Comparison of sagittal spinopelvic parameters in different types of double-level degenerative lumbar spondylolisthesis: a retrospective study}

guangzhi zhang

lanzhou university second hospital https://orcid.org/0000-0003-3193-0297

\section{Yajun Deng}

Lanzhou University Second Hospital

\section{Xuegang He}

Lanzhou University Second Hospital

\section{Enhui Ren}

Lanzhou University Second Hospital

\section{Zuolong Wu}

Lanzhou University Second Hospital

\section{Fengguang Yang}

Lanzhou University Second Hospital

\section{Liang Yang}

Lanzhou University Second Hospital

\section{Zhanjun Ma}

Lanzhou University Second Hospital

\section{Yichen Gao}

Lanzhou University Second Hospital

\section{Xudong Guo}

Lanzhou University Second Hospital

\section{Yidian Wang}

Lanzhou University Second Hospital

\section{Mingqiang Liu}

Lanzhou University Second Hospital

Daxue Zhu

Lanzhou University Second Hospital

Xuewen Kang ( $\nabla$ ery_kangxw@lzu.edu.cn )

Research article 
Keywords: Degenerative lumber spondylolisthesis, Double-level spondylolisthesis, Sagittal spinopelvic parameters

Posted Date: June 2nd, 2020

DOI: https://doi.org/10.21203/rs.3.rs-30670/v1

License: (c) (i) This work is licensed under a Creative Commons Attribution 4.0 International License. Read Full License 


\section{Abstract \\ Background}

Degenerative lumber spondylolisthesis (DLS), a common orthopaedic disease causing low back pain, seriously affects daily life and work. Although sagittal spinopelvic parameters have been studied in single-level DLS patients, investigations into different types of double-level DLS are scarce. This study aims to analyse the demographic and radiological parameters of patients with different types of doublelevel DLS and compare their differences to provide a reference for guiding surgical treatment and restoring sagittal balance of DLS patients.

\section{Methods}

From January 2014 to January 2020, double-level DLS patients' records were retrospectively reviewed and divided into three types: anterior, posterior, and combined; the anterior and combined types were studied. Two spin surgeons measured the sagittal spinopelvic parameters: $\mathrm{C} 7 \mathrm{tilt}$, maximal thoracic kyphosis (TKmax), maximal lumbar lordosis (LLmax), pelvic incidence (PI), pelvic tilt (PT), and sacral slope (SS). Following descriptive analysis, demographic and radiographic data were compared between the anterior and combined types.

\section{Results}

Patients in the anterior type group $(n=40)$ were older $(67.68 \pm 6.66$ vs. $61.72 \pm 10.06$ years, $P=0.031)$, and the proportion of females were significantly higher $(90 \%$ vs. $50 \%, P=0.001)$ than the combined group $(n=18)$. Both groups had different levels of chronic lower back pain, but the incidence of radiating leg pain and neurogenic claudication was significantly higher in the anterior type. Owestry disability index and visual analogue scale lower back scores were also higher in the anterior type. In the anterior type, $\mathrm{C7}$ tilt (7.14 \pm 2.15 vs. $5.41 \pm 2.28, P=0.007)$, LLmax ( $50.02 \pm 14.76$ vs. $36.96 \pm 14.56, P=0.003), \mathrm{PI}(68.28 \pm$ 9.16 vs. $55.53 \pm 14.19, P<0.001)$, PT ( $28.68 \pm 7.31$ vs. $19.38 \pm 4.70, P<0.001)$, and PT/PI $(42.45 \pm 11.22$ vs. $36.04 \pm 9.87, P=0.041)$ were significantly higher. In the anterior type, PI correlated significantly positively with LLmax $(r=0.59)$ and SS $(r=0.71)$. LLmax and SS $(r=0.65)$ had a positive correlation. PT/PI and SS $(r=-0.77)$ had a negative correlation. In the combined type, PI correlated positively with LLmax $(r=0.61)$ and SS $(r=0.88)$, and PT/PI correlated negatively with SS $(r=-0.81)$.

\section{Conclusions}

In patients with double-level DLS, the sagittal spinopelvic parameters differ between the anterior and combined types. PI and LLmax were significantly higher in the anterior type, resulting in compensation by forward flexion and pelvic retroversion. Spinal surgeons should focus on correcting sagittal deformities, 
relieving postoperative clinical symptoms, and improving quality of life during fusion surgery, which is proven to guarantee long-term surgical results.

\section{Background}

Degenerative lumber spondylolisthesis (DLS) is a common degenerative spinal disease. It is defined by the tendency of the superior vertebra to slide forward or backward relative to an adjacent inferior vertebra in the sagittal plane without accompanying isthmic fissures. DLS is most common in L4-L5 segment, and is more common in people aged 50-60 years and in women [1,2]. The aetiology and pathogenesis of DLS is quite complicated, and a variety of factors cause the adjacent vertebral body slippage that produces a series of syndromes such as low back pain, radiating leg pain, neurogenic claudication, numbness, weakness, and intermittent claudication [3]. Many studies have shown that DLS is usually accompanied by diseases such as arthritis, disc degeneration, and spinal stenosis [4-6]. As the spondylolisthesis progresses, the spine and pelvis morphology of patients undergoes compensatory changes to maintain spine balance, and the sagittal spinopelvic parameters change accordingly [7-9].

In 1992, Duval-Beaupère et al. [10] defined pelvic parameters and described a morphological parameter, namely pelvic incidence (PI), and two position parameters, namely pelvic tilt (PT) and sacral slope (SS). The spine parameters mainly include thoracic kyphosis (TK), lumbar lordosis (LL) and C7 tilt [11]. The spine and pelvis are connected in an anatomical structure, and the sagittal parameters between them are related and coordinated. Hitherto, the importance of these sagittal parameters has been studied extensively and thoroughly in single-level DLS $[1,8,12,13]$. However, there are only few studies on spinopelvic parameters in patients with double-level DLS.

Emmanuelle et al. [14] studied the sagittal spinopelvic parameters of double-level and single-level DLS. The results showed that the double-level DLS had larger PI and C7 tilt, and it initiated the compensation mechanism such as forward flexion and pelvic retroversion. Iguchi and Wakami et al. [15] divided the double-level DLS into three types: anterior type-anterolisthesis of two neighbouring vertebral bodies;posterior type-retrolisthesis of two neighbouring vertebral bodies; and combined typecombination of anterolisthesis (usually seen in lower vertebral body) and anterolisthesis (upper vertebral body) (Fig. 1). However, to the best of our knowledge, no one has studied different types of DLS. Therefore, analysing the sagittal spinopelvic parameters with different types of DLS patients can be used as a symptomatic assessment or treatment target, which helps to better understand this disease and enable patients to get better treatment.

The purpose of this study is to analyse the demographic and radiological parameters of patients with the anterior type and combined type of double-level DLS and compare the changes in these parameters between the two types of double-level DLS to understand better the mechanism of the occurrence and development of this disease and formulate a reference for surgical treatment plan.

\section{Methods}




\section{Patients' selection}

From January 2014 to January 2020, data on 58 double-level DLS patients who needed surgical treatment were collected from the Spinal Surgery Department of the Lanzhou University Second Hospital. Inclusion criteria were as follows: (1) any adult patient above 18 years of age; (2) anterior type and combined type of double-level DLS patients; (3) presence of complete radiological data of the full spinal standing position including the femoral heads. Exclusion criteria were as follows: (1) posterior type of double-level DLS patients (the incidence of this type is very low and clinically rare. Two cases of this type were collected but clinical data is incomplete; they were therefore excluded); (2) isthmic spondylolisthesis; (3) previous history of surgery or trauma; and (4) presence of other spinal diseases including tumours, tuberculosis, infection, scoliosis, kyphosis, and metabolic bone disease.

\section{Data collection}

Collected data included standard demographic information (age, sex, body mass index, BMI), slippage classification (using Meyerding classification method), main clinical symptoms (including chronic low back pain, radiating leg pain, neurogenic claudication, numbness, and weakness), clinical outcomes based on quality of life assessment (including ODI scores, VAS low back scores, and VAS leg pain scores) and radiographic parameters All radiographic data were measured from the picture archiving and communication system (PACS) by two senior spine surgeons. The spondylolisthesis distance and sagittal spinopelvic parameters were measured on the full spinal standing lateral position using $\mathrm{x}$-ray projections including the femoral heads. The slippage distance was measured as distance that the superior vertebra slides forward or backward relative to an adjacent inferior vertebra in the sagittal plane [10, 14] (Fig. 2A). The sagittal spinopelvic parameters are as follows (Fig. 2B): (1) C7 tilt: angle formed by the line between the centre of the $\mathrm{C} 7$ vertebral body and the midpoint of the upper sacral endplate and the vertical reference line, which are used to evaluate the overall sagittal plane balance of the spine; (2) maximal thoracic kyphosis (TKmax): angle between the upper endplate of T1 vertebral body and the lower endplate of T12 vertebral body; (3) maximal lumbar lordosis (LLmax): the angle between the upper endplate of $\mathrm{L} 1$ vertebral body and the lower endplate of $\mathrm{L} 5$ vertebral body; (4) pelvic incidence ( $\mathrm{PI})$ : angle between the line connecting the midpoint of the upper sacrum endplate with the centre of the bilateral femoral head and perpendicular to the upper sacral endplate tangent; (5) pelvic tilt (PT): angle between the line connecting the midpoint of the upper sacral endplate and the centre of the bilateral femoral head and the horizontal perpendicular line; (6) sacral slope (SS): angle between the upper endplate of the sacrum and the horizontal line. In addition, to evaluate the relationship between the sagittal spinopelvic parameters and $\mathrm{PI}$, we calculated the ratio of LLmax to PI (LLmax/PI) and the ratio of PT to PI (PT/PI), which are the morphological parameters unique to everyone [14].

\section{Statistical analysis}

Statistical analysis was performed using IBM SPSS Statistics version 23. A Shapiro-Wilk test was used to evaluate the data distribution. All continuous variables were described using means and standard deviations, and an independent sample t test was used for comparison between the two groups. 
Frequency analysis was used to describe the categorical variables, and comparison between the two groups using $\chi 2$ test. Pearson correlation analysis was used to evaluate the correlation between different parameters, and $P<0.05$ was considered statistically significant.

\section{Results}

\section{Demographic data}

Altogether, 58 patients with DLS were included in this study (13 males and 45 females, with an average age of $65.83 \pm 8.26$ years), including 40 patients in the anterior type $(69.0 \%)$ and 18 patients in the combined type (31\%). Patients in the anterior type were older compared to those in the combined type ( $67.68 \pm 6.66$ vs. $61.72 \pm 10.06, P=0.031)$. The proportion of females was higher in the anterior type (90\% vs $50 \%, P=0.001)$. Meyerding I slips were more common in the anterior type $(70.7 \%)$, while the incidence of Meyerding I and II were comparable in the combined type. The main clinical symptoms showed that the patients in both groups had varying degrees of chronic low back pain. The incidence of radiating leg pain and neurogenic claudication in the anterior type was significantly higher than that in the combined type. The incidence of numbness and weakness in the two groups of patients was quite. The results of the quality of life evaluation showed that the ODI scores and VAS low back scores of the anterior type were significantly higher than those of combined type were, while the VAS leg pain scores had no significant difference between the two groups. There was no significant difference in BMI between the two groups (Table 1).

\section{Radiographic data}

In both groups, the degree of slippage was greater in L4L5 and L5S1. Compared with the anterior type, patients in the combined type had greater levels of L3L4 slippage ( $7.58 \pm 1.39$ vs. $8.84 \pm 0.52, P=0.001)$. The L4L5 and L5S1 levels slippage had no statistical significance between the two groups. No cases of L2L3 level slippage was found in the anterior type (Table 2).

A comparison of the anterior type patients with the combined type revealed that C7 tilt $(7.14 \pm 2.15 \mathrm{vs}$. $5.41 \pm 2.28, P=0.007), \mathrm{PI}(68.28 \pm 9.16$ vs. $55.53 \pm 14.19, P \otimes 0.001)$, LLmax (50.02 \pm 14.76 vs. $36.96 \pm$ $14.56, P=0.003), \mathrm{PT}(28.68 \pm 7.31$ vs. $29.99 \pm 6.67, P<0.001)$ and PT/PI ( $42.45 \pm 11.22$ vs. $36.04 \pm 9.78$, $P=0.041)$ was significantly greater in the anterior type group. The TKmax, SS, and LLmax/PI were not significantly different between the two groups (Table 3).

There were significant correlations between the sagittal spinopelvic parameters in the two groups. In the anterior type group, PI was positively correlated with $\operatorname{LLmax}(r=0.59, P<0.001)$ and SS $(r=0.71, P<$ $0.001)$. Additionally, LLmax was positively correlated with $S S(r=0.65, P<0.001)$. PT/PI was negatively correlated with SS $(r=-0.77, P<0.001)$ and LLmax $(r=-0.59, P<0.001)$ (Table 4). In the combined type group, PI was positively correlated with $\operatorname{LLmax}(r=0.61, P=0.007), \mathrm{PT}(r=0.48, P=0.043)$ and $\mathrm{SS}(\mathrm{r}=$ $0.88, P<0.001)$. Additionally, LLmax was positively correlated with $S S(r=0.79, P<0.001)$. The PT/PI was negatively correlated with SS $(r=-0.81, P<0.001)$ and LLmax $(r=-0.81, P<0.001)$ (Table 5). 


\section{Discussion}

The normal spine exhibits an S-shaped physiological curvature, and the spine and pelvis jointly maintain trunk balance. Owing to the development of DLS, compensatory changes in spine and pelvis occurred to maintain trunk balance. The sagittal spinopelvic parameters can adequately reflect this balance and compensate for biomechanical changes of the spine after DLS $[16,17]$. To the best of our knowledge, this study is the first report of the differences and correlations between spinopelvic parameters of different types of DLS patients.

In DLS, the female sex has been considered a risk factor for spondylolisthesis. In this study, $77.6 \%$ of double-level DLS patients were women, which is similar to that obtained by previous studies $[18,19]$. Previous studies have shown that compared with single-level DLS, women with double-level DLS account for a larger proportion (approximately 70\%) [14]. Interestingly, this study found that women in the anterior type accounted for a larger proportion of women in double-level DLS, while men and women had a similar proportion in the combined type group. Compared with the combined type, the anterior type group patients were older (over 69 years old), suggesting that these patients may gradually develop double-level spondylolisthesis owing to previous delayed treatment single-level spondylolisthesis. However, further research is still needed to confirm this process. A study has shown that the age of DLS patients is an important consideration factor for surgery because they may be associated with more comorbidities and a higher risk of postoperative complications [20]. However, other studies suggest that compared with young DLS patients, elderly patients have significantly improved postoperative symptoms, and there is no significant increase in complications and mortality [21, 22]. It can be seen that for elder DLS patients, surgical treatment has obvious advantages. In addition, many studies have showed that the lumbar intervertebral instability and nerve compression are the primary factors leading to low back pain and functional disabilities in DLS patients [23-25]. This study found that patients in both groups had varying degrees of chronic low back pain. The incidence of radiating leg pain and neurogenic claudication in the anterior type was significantly higher. The preoperative ODI and VAS scores showed that the quality of life of the anterior type patients was significantly lower and VAS low back scores was higher than those in the combined type. The above results indicate that the lumbar intervertebral instability is more serious in the anterior type and leads to more severe clinical symptoms and a significant reduction in quality of life. Therefore, for such patients, when making surgical plans, enhancing the stability of the spine, relieving the postoperative clinical symptoms, and improving the quality of life after surgery may be important considerations for surgical treatment.

Previous studies on spinopelvic parameters in DLS patients have been reported in the literature [26-28]. The most common level of slippage in single-level DLS patients is L4L5, but the levels of L3L4-L4L5 and L4L5-L5S1 is common in double-level DLS patients [14]. This study found that L3L4-L4L5 and L4L5-L5S1 levels of spondylolisthesis are common in double-level DLS, which is similar to that obtained in previous studies. Hitherto, few studies have been conducted on the characteristics of spinopelvic parameters in double-level DLS patients. Iguchi et al. [15] divided double-level DLS into three types (anterior type, posterior type and combined type), and studied pedicle-facet angles,facet joint shapeland vertebra 
rotation direction; they found that the sagittal facet joint angle and W-shaped facet joint are the key factors for the vertebral body forward slip. Ferrero et al. [7] showed that compared with single-level DLS, C7 tilt, PI and PT were significantly higher in patients with double-level DLS, but LLmax was significantly lower, indicating that sagittal imbalance was more serious, lumbar lordosis was severely lost, and more compensatory mechanisms (such as forward flexion and pelvic retroversion) were activated in doublelevel DLS. However, they did not analyse the difference between the spinopelvic parameters of different types of double-level DLS patients. The present study found that C7 tilt, LLmax, PI and PT increased significantly in the anterior type. These results all suggest that a higher PI in the anterior type may be a risk factor for anterolisthesis of two adjacent vertebral bodies. As the spondylolisthesis progresses, LLmax gradually increases. The change in lumbar lordosis is accompanied by the inclination of the sacrum, and the SS changes accordingly. Because PT and SS have the same effect in evaluating the pelvic shape, according to the geometric relationship PI = PT + SS, the PT increased, and to maintain the sagittal balance of the spine, C7 tilt increased [29-31]. As the LLmax of increases, changes in thoracolumbar segment also easily cause lumbar lordosis changes, which in turn accelerate lumbar degeneration [32]. The combined type group may compensate each other because of antrolisthesis and retrolisthesis. Thus, the parameters C7 tilt, LLmax, PI, and PT are smaller than those of the anterior type, which may be one of the reasons why combined type patients have milder clinical symptoms and better quality of life than that of anterior type patients. To maintain the sagittal balance of the spine $\square L L m a x$ increased in DLS with angular changes in TKmax. However, owing to the relatively fixed thoracic spine and low mobility, the compensatory changes is not significant in elderly DLS patients. Hence, there was no significant difference in TKmax between the two groups. The ratios LLmax/PI and PT/PI are used to evaluate the relationship between spinopelvic parameters and $\mathrm{PI}$, which is a morphological parameter unique to everyone [33]. The ratio $\mathrm{LL} / \mathrm{PI}$, as a spinopelvic parameter, is related to the occurrence and development of proximal junctional degenerations [34].This study found that the LLmax/PI ratio imbalance in the anterior type group patients was more obvious, further indicating that the anterior type may be due to the evolution of a single-level slippage. Unfortunately, probably because of the small sample size in this study, the ratio LLmax/PI in the two groups were not statistically significant. For combined type patients, spondylolisthesis may not be caused by high PI, but the imbalance of LLmax/PI ratio after spondylolisthesis causes another vertebral body to slip backward to maintain the body's balance. Previous studies have shown that PI has a positive correlation with $L L$, and LL has a linear correlation with PT and SS, whereas PT has a linear correlation with LLmax/PI [14]. In this study, there was a significant correlation between the spinopelvic parameters in the two groups. In the anterior type group, PI was positively correlated with LLmax, and LLmax and SS have a positive correlation. In the combined type group, LLmax was positively correlated with PI and SS, and LLmax/PI was positively correlated with SS.

Our study had several limitations. First, the low incidence of double-level DLS results in a small sample size, which may have a statistical impact. Secondly, this is a retrospective analysis, which is subject to some restrictions in data collection. The radiological data in this study are the results of preoperative examination; there were no intraoperative data and postoperative follow-up data, thus there was no study 
of postoperative spinopelvic parameters and patients' quality of life. Finally, no complete and sufficient posterior type patients' data were collected in this study. In the future, we plan to cooperate with other hospitals to conduct multi-centre clinical research, expand the sample size, and further study multi-centre data to verify our research results.

\section{Conclusion}

In recent years, the problem of sagittal balance during the reconstruction of spinopelvic alignment in DLS patients has received increasing attention. In patients with double-level DLS, the spinopelvic parameters are different between the anterior type and combined type. Compared with the combined type group, PI and LLmax were significantly higher in the anterior type and, simultaneously, the compensation mechanism such as forward flexion and pelvic tilt was activated. Decompression and fusion are effective ways to treat this disease. For surgical treatments, surgeons should pay attention to the effect of different types of double-level DLS on spinopelvic parameters. With respect to the more severe clinical symptoms, worse quality of life, and more severe sagittal imbalance in the anterior type group, spinal surgeons should focus on correcting sagittal deformities, relieving postoperative clinical symptoms, and improving quality of life during fusion surgery, which has been proven to guarantee long-term surgical results.

\section{Abbreviations}

DLS

Degenerative lumber spondylolisthesis

PACS

The picture archiving and communication system

BMI

Body mass index

SD

Standard deviation

ODI

Owestry disability index

VAS

Visual analogue scale

SD

Standard deviation

TKmax

Maximal thoracic kyphosis

LLmax

Maximal lumbar lordosis

$\mathrm{PI}$ 
Pelvic incidence,

PT

Pelvic tilt

SS

Sacral slope

\section{Declarations}

\section{Ethics approval and consent to participate}

Ethical approval was obtained from the Lanzhou University Second Hospital Medical Ethics Committee.

Written informed consent was provided by all patients prior to involvement in the study.

\section{Consent for publication}

Written informed consent for publication was obtained from all participants

\section{Availability of data and materials}

All data are true and correct, and all patients were hospitalised in our hospital.

\section{Competing interests}

The authors declare that they have no competing interests.

\section{Funding}

This work was financially supported by the Lanzhou University Innovation and Entrepreneurship Cultivation Project (cxcy201906), Doctoral Research Fund of Lanzhou University Second Hospital (YJSBD-09), Chinese Medicine Administration Research Project of Gansu province (GZK-2019-46), and Cuiying Technology Innovation Project of Lanzhou University Second Hospital (CY2019-MS10).

\section{Authors' contributions}

XWK, GZZ, and YJD conceived and designed the study. XWK guided the project. XDG, YDW, MQL, ZLW, FGY, LY and DXZ contributed to data collection. XGH, HER, ZJM and YCG performed statistical analyses. GZZ, YJD, XGH and XWK wrote and revised the manuscript. All authors read and approved the final manuscript.

\section{Acknowledgments}


We are grateful to the international cooperation base of Gansu Province for the research on pain in spinal disorders, and to the key laboratory of Orthopaedics Disease of Gansu Province, Lanzhou University, Lanzhou, China. We would like to thank Editage (www.editage.cn) for English language editing.

\section{References}

1. Bydon M, Alvi MA, Goyal A. Degenerative Lumbar Spondylolisthesis: Definition, Natural History, Conservative Management, and Surgical Treatment. Neurosurg Clin N Am. 2019;30:299-304. https://doi.org/10.1016/j.nec.2019.02.003.

2. Sun K, Liang L, Yin H, Yu J, Feng M, Zhan J, Jin Z, Yin X, Wei X, Zhu L. Manipulation for treatment of degenerative lumbar spondylolisthesis: A protocol of systematic review and meta-analysis. Medicine. 2019;98(49):e18135. https://doi.org/10.1097/MD.0000000000018135.

3. Ilyas H, Udo-Inyang IJ, Savage J. Lumbar Spinal Stenosis and Degenerative Spondylolisthesis: A Review of the SPORT Literature. Clin Spine Surg. 2019;32:272-8. https://doi.org/10.1097/BSD.0000000000000841.

4. Huang K, Hsu Y, Chen W, Tsai H, Yan J, Wang J, Liu W, Lin R. The roles of IL-19 and IL-20 in the inflammation of degenerative lumbar spondylolisthesis. J Inflamm (Lond). 2018;15:19. https://doi.org/10.1186/s12950-018-0195-6.

5. Hiyama A, Katoh H, Sakai D, Tanaka M, Sato M, Watanabe M. The correlation analysis between sagittal alignment and cross-sectional area of paraspinal muscle in patients with lumbar spinal stenosis and degenerative spondylolisthesis. BMC Musculoskel Dis. 2019;20:352. https://doi.org/10.1186/s12891-019-2733-7.

6. Ramhmdani S, Ishida W, Perdomo-Pantoja A, Witham TF, Lo SL, Bydon A. Synovial Cyst as a Marker for Lumbar Instability: A Systematic Review and Meta-Analysis. World Neurosurg. 2019;122:e105968. https://doi.org/10.1016/j.wneu.2018.10.228.

7. Ferrero E, Ould-Slimane M, Gille O, Guigui P, French SSS. Sagittal spinopelvic alignment in 654 degenerative spondylolisthesis. Eur Spine J. 2015;24:1219-27. https://doi.org/10.1007/s00586-0153778-4.

8. Lai Q, Gao T, Lv X, Liu X, Wan Z, Dai M, Zhang B, Nie T. Correlation between the sagittal spinopelvic alignment and degenerative lumbar spondylolisthesis: a retrospective study. BMC Musculoskel Dis. 2018;19:151. https://doi.org/10.1186/s12891-018-2073-z.

9. Nakamae T, Nakanishi K, Kamei N, Adachi N. The correlation between sagittal spinopelvic alignment and degree of lumbar degenerative spondylolisthesis. J Orthop Sci. 2019;24:969-73. https://doi.org/10.1016/j.jos.2019.08.021.

10. Duval-Beaupère G, Schmidt C, Cosson P. A Barycentremetric study of the sagittal shape of spine and pelvis: the conditions required for an economic standing position. Ann Biomed Eng. 1992;20:451-62. https://doi.org/10.1007/bf02368136. 
11. Ding Y, Liu B, Qiao H, Yin L, He W, Si F, Wang D. Can knee flexion contracture affect cervical alignment and neck tension? A prospective self-controlled pilot study. Spine J. 2020;20:251-60. https://doi.org/10.1016/j.spinee.2019.09.008.

12. Kim MK, Lee SH, Kim ES, Eoh W, Chung SS, Lee CS. The impact of sagittal balance on clinical results after posterior interbody fusion for patients with degenerative spondylolisthesis: a pilot study. BMC Musculoskel Dis. 2011;12:69. https://doi.org/10.1186/1471-2474-12-69.

13. Chuang CY, Liaw MY, Wang LY, Huang YC, Pong YP, Chen CW, Wu RW, Lau YC. Spino-pelvic alignment, balance, and functional disability in patients with low-grade degenerative lumbar spondylolisthesis. J Rehabil Med. 2018;50:898-907. https://doi.org/10.2340/16501977-2489.

14. Ferrero E, Simon A, Magrino B, Ould-Slimane M, Guigui P. Double-level degenerative spondylolisthesis: what is different in the sagittal plane? Eur Spine J. 2016;25:2546-52. https://doi.org/10.1007/s00586-016-4384-9.

15. Iguchi T, Wakami T, Kurihara A, Kasahara K, Yoshiya S, Nishida K. Lumbar multilevel degenerative spondylolisthesis: radiological evaluation and factors related to anterolisthesis and retrolisthesis. $J$ Spinal Disord Tech. 2002;15:93-9. https://doi.org/10.1097/00024720-200204000-00001.

16. Li J, Wen Z, He X, Wei X. Advances on the study of movable artificial vertebral body. Zhongguo Gu Shang. 2019;32:677-82. https://doi.org/10.3969/j.issn.1003-0034.2019.07.017.

17. Mehta VA, Amin A, Omeis I, Gokaslan ZL, Gottfried ON. (2015) Implications of spinopelvic alignment for the spine surgeon. Neurosurgery 76 Suppl 1:42-56. https://doi.org/10.1227/01.neu.0000462077.50830.1a.

18. Aono K, Kobayashi T, Jimbo S, Atsuta Y, Matsuno T. Radiographic analysis of newly developed degenerative spondylolisthesis in a mean twelve-year prospective study. Spine. 2010;35:887-91. https://doi.org/10.1097/BRS.0b013e3181cdd1aa.

19. Ferrero E, Ould-Slimane M, Gille O, Guigui P, French SSS. Sagittal spinopelvic alignment in 654 degenerative spondylolisthesis. Eur Spine J. 2015;24:1219-27. https://doi.org/10.1007/s00586-0153778-4.

20. Blamoutier A, Guigui P, Charosky S, Roussouly P, Chopin D, Groupe DÉDL. Surgery of lumbar and thoracolumbar scolioses in adults over 50 . Morbidity and survival in a multicenter retrospective cohort of 180 patients with a mean follow-up of 4.5 years. Orthop Traumatol Surg Res. 2012;98:528-35. https://doi.org/10.1016/j.otsr.2012.04.014.

21. Rihn JA, Hilibrand AS, Zhao W, Lurie JD, Vaccaro AR, Albert TJ, Weinstein J. Effectiveness of surgery for lumbar stenosis and degenerative spondylolisthesis in the octogenarian population: analysis of the Spine Patient Outcomes Research Trial (SPORT) data. J Bone Joint Surg Am. 2015;97:177-85. https://doi.org/10.2106/JBJS.N.00313.

22. Rothenfluh DA, Koenig M, Stokes OM, Behrbalk E, Boszczyk BM. (2014) Access-related complications in anterior lumbar surgery in patients over 60 years of age. Eur Spine J 23 Suppl 1:86-92. https://doi.org/10.1007/s00586-014-3211-4. 
23. Fritz JM, Delitto A, Welch WC, Erhard RE. Lumbar spinal stenosis: a review of current concepts in evaluation, management, and outcome measurements. Arch Phys Med Rehabil. 1998;79:700-8. https://doi.org/10.1016/s0003-9993(98)90048-x.

24. Huang KY, Lin RM, Lee YL, Li JD. Factors affecting disability and physical function in degenerative lumbar spondylolisthesis of L4-5: evaluation with axially loaded MRI. Eur Spine J. 2009;18:1851-7. https://doi.org/10.1007/s00586-009-1059-9.

25. Chen SY, Lu ML, Niu CC, Tsai TT, Liao JC, Chen LH, Chen WJ. Results of instrumented posterolateral fusion in treatment of lumbar spondylolisthesis with and without segmental kyphosis: A retrospective investigation. Biomed J. 2015;38:262-8. https://doi.org/10.4103/2319-4170.145768.

26. Samuel AM, Moore HG, Cunningham ME. Treatment for Degenerative Lumbar Spondylolisthesis: Current Concepts and New Evidence. Curr Rev Musculoskelet Med. 2017;10:521-9. https://doi.org/10.1007/s12178-017-9442-3.

27. Uysal M, Ozalay M, Derincek A, Kochai A, Turker M. Effect of PLIF and TLIF on sagittal spinopelvic balance of patients with degenerative spondylolisthesis. Acta Orthop Traumato. 2018;52:272-6. https://doi.org/10.1016/j.aott.2018.03.001.

28. Ha J, Hwang CJ, Lee D, Lee MY, Yoon SJ, Lee CS. Spinopelvic parameters in degenerative spondylolisthesis. Acta Orthop Belg. 2019;85:253-9.

29. Celestre PC, Dimar JRN, Glassman SD. Spinopelvic Parameters: Lumbar Lordosis, Pelvic Incidence, Pelvic Tilt, and Sacral Slope: What Does a Spine Surgeon Need to Know to Plan a Lumbar Deformity Correction? Neurosurg Clin N Am. 2018;29:323-9. https://doi.org/10.1016/j.nec.2018.03.003.

30. Radovanovic I, Urquhart JC, Ganapathy V, Siddiqi F, Gurr KR, Bailey SI, Bailey CS. Influence of postoperative sagittal balance and spinopelvic parameters on the outcome of patients surgically treated for degenerative lumbar spondylolisthesis. J Neurosurg Spine. 2017;26:448-53. https://doi.org/10.3171/2016.9.SPINE1680.

31. Lim JK, Kim SM. Comparison of Sagittal Spinopelvic Alignment between Lumbar Degenerative Spondylolisthesis and Degenerative Spinal Stenosis. J Korean Neurosurg Soc. 2014;55:331-6. https://doi.org/10.3340/jkns.2014.55.6.331.

32. Hayashi K, Toyoda H, Terai H, Suzuki A, Hoshino M, Tamai K, Ohyama S, Nakamura H. Cervical lordotic alignment following posterior spinal fusion for adolescent idiopathic scoliosis: reciprocal changes and risk factors for malalignment. Journal of neurosurgery. J Neurosurg Pediatr. 2017;19:440-7. https://doi.org/10.3171/2016.9.PEDS16298.

33. Mac-Thiong J, Roussouly P, Berthonnaud E, Guigui P. Age- and sex-related variations in sagittal sacropelvic morphology and balance in asymptomatic adults. Eur Spine J 20 Suppl. 2011;5:572-7. https://doi.org/10.1007/s00586-011-1923-2.

34. Galla F, Wähnert D, Liljenqvist U. Georg Schmorl Prize of the German Spine Society (DWG) 2017: correction of spino-pelvic alignment with relordosing mono- and bisegmental TLIF spondylodesis. Eur Spine J. 2018;27:789-96. https://doi.org/10.1007/s00586-018-5503-6. 


\section{Tables}

Table 1 Demographic data comparison

\begin{tabular}{|llllll|}
\hline & \multicolumn{4}{l}{ Anterior type $(\mathrm{n}=40)$} & \multicolumn{2}{c}{ Combined type $(\mathrm{n}=18)$} & P value \\
& Mean & SD & Mean & SD & \\
\hline Age(years) & 67.68 & 6.66 & 61.72 & 10.06 & 0.031 \\
\hline Female(\%) & 90 & - & 50 & - & 0.001 \\
\hline BMI(kg/m $\left.{ }^{2}\right)$ & 27.23 & 2.93 & 26.79 & 3.73 & 0.629 \\
\hline Spondylolisthesis grade(I/II) & $31 / 9$ & - & $9 / 9$ & - & 0.036 \\
\hline Main symptoms(\%) & & & & & \\
\hline Chronic low back pain & 100 & - & 94.4 & - & 0.679 \\
\hline Radiating leg pain & 87.5 & - & 55.6 & - & 0.018 \\
\hline Neurogenic claudication & 77.5 & - & 50 & - & 0.036 \\
\hline Numbness & 65.0 & - & 55.6 & - & 0.493 \\
\hline Weakness & 27.5 & - & 22.2 & - & 0.920 \\
\hline Quality of life assessment & & & & & \\
\hline ODI scores & 60.60 & 8.50 & 50.44 & 5.43 & 0.000 \\
\hline VAS low back scores & 6.53 & 0.75 & 5.94 & 0.73 & 0.008 \\
\hline VAS leg pain scores & 6.45 & 0.88 & 6.50 & 0.71 & 0.832 \\
\hline BMl: body mass index; SD: standard deviation; ODI: owestry disability index; VAS: visual analog scale \\
\hline
\end{tabular}

\begin{tabular}{|c|c|c|c|c|c|}
\hline \multicolumn{6}{|c|}{ Table 2 Comparison of the slippage for each lumbar level } \\
\hline & \multicolumn{2}{|c|}{ Anterior type $(n=40)$} & \multicolumn{2}{|c|}{ Combined type $(n=18)$} & \multirow[t]{2}{*}{$P$ value } \\
\hline & Mean & SD & Mean & SD & \\
\hline L2L3 & - & - & 7.49 & 0.58 & - \\
\hline L3L4 & 7.58 & 1.39 & 8.84 & 0.52 & 0.001 \\
\hline L4L5 & 9.36 & 1.93 & 11.33 & 0.75 & 0.089 \\
\hline L5S1 & 12.44 & 3.69 & 11.73 & 2.00 & 0.495 \\
\hline
\end{tabular}


Table 3 Comparison of radiological parameters

\begin{tabular}{|c|c|c|c|c|c|}
\hline \multirow[t]{2}{*}{ Parameters } & \multicolumn{2}{|c|}{ Anterior type $(n=40)$} & \multicolumn{2}{|c|}{ Combined type $(\mathrm{n}=18)$} & \multirow[t]{2}{*}{$P$ value } \\
\hline & Mean & SD & Mean & SD & \\
\hline C7 tilt $\left({ }^{\circ}\right)$ & 7.14 & 2.15 & 5.41 & 2.28 & 0.007 \\
\hline $\operatorname{TKmax}\left({ }^{\circ}\right)$ & 39.49 & 4.84 & 38.87 & 4.50 & 0.649 \\
\hline $\operatorname{LLmax}\left({ }^{\circ}\right)$ & 50.02 & 14.76 & 36.96 & 14.56 & 0.003 \\
\hline $\mathrm{PI}\left({ }^{\circ}\right)$ & 68.28 & 9.16 & 55.53 & 14.19 & 0.000 \\
\hline $\mathrm{PT}\left({ }^{\circ}\right)$ & 28.68 & 7.31 & 19.38 & 4.70 & 0.000 \\
\hline SS $\left(^{\circ}\right)$ & 38.68 & 10.40 & 36.49 & 11.42 & 0.474 \\
\hline PT/PI (\%) & 42.45 & 11.22 & 36.04 & 9.87 & 0.041 \\
\hline LLmax/PI (\%) & 72.75 & 16.69 & 65.80 & 21.45 & 0.234 \\
\hline \multicolumn{6}{|c|}{$\begin{array}{l}\text { SD notes for standard deviation, TKmax for maximal thoracic kyphosis, LLmax for maximal lumbar } \\
\text { lordosis, PI for pelvic incidence, PT for pelvic tilt, SS for sacral slope, PT/PI for the ratio of pelvic tilt } \\
\text { divided by pelvic incidence, LLmax/PI for the ratio of maximal lumbar lordosis divided by pelvic } \\
\text { incidence }\end{array}$} \\
\hline
\end{tabular}

Table 4

Results of Pearson correlation analysis between radiographic parameters in anterior type patients

\begin{tabular}{|c|c|c|c|c|c|c|c|c|}
\hline & C7 tilt & TKmax & LLmax & PI & PT & SS & PT/PI & LLmax/PI \\
\hline C7 tilt & 1.00 & & & & & & & \\
\hline TKmax & -0.05 & 1.00 & & & & & & \\
\hline LLmax & 0.08 & -0.14 & 1.00 & & & & & \\
\hline PI & 0.33 & -0.19 & 0.59 & 1.00 & & & & \\
\hline PT & 0.36 & -0.13 & -0.27 & 0.25 & 1.00 & & & \\
\hline SS & -0.03 & -0.10 & 0.65 & 0.71 & -0.39 & 1.00 & & \\
\hline PT/PI & 0.17 & -0.01 & -0.59 & -0.31 & 0.84 & -0.77 & 1.00 & \\
\hline LLmax/PI & -0.06 & -0.06 & 0.92 & 0.23 & -0.48 & 0.46 & -0.59 & 1.00 \\
\hline
\end{tabular}


Table 5

Results of Pearson correlation analysis between radiographic parameters in combined type patients

\begin{tabular}{|c|c|c|c|c|c|c|c|c|}
\hline & C7 tilt & TKmax & LLmax & PI & PT & SS & PT/PI & LL/PImax \\
\hline C7 tilt & 1.00 & & & & & & & \\
\hline TKmax & -0.26 & 1.00 & & & & & & \\
\hline LLmax & 0.11 & -0.33 & 1.00 & & & & & \\
\hline PI & -0.28 & -0.29 & 0.61 & 1.00 & & & & \\
\hline PT & -0.30 & 0.03 & -0.20 & 0.48 & 1.00 & & & \\
\hline SS & -0.11 & -0.37 & 0.79 & 0.88 & 0.03 & 1.00 & & \\
\hline PT/PI & -0.05 & 0.31 & -0.81 & -0.48 & 0.54 & -0.81 & 1.00 & \\
\hline LLmax/PI & 0.31 & -0.25 & 0.87 & 0.15 & -0.56 & 0.46 & -0.73 & 1.00 \\
\hline $\begin{array}{l}\text { TKmax not } \\
\text { incidence, } \\
\text { incidence, L }\end{array}$ & $\begin{array}{l}\text { or maxi } \\
\text { or pelvi } \\
\text { ax/PI f }\end{array}$ & $\begin{array}{l}\text { I thoracic } \\
\text { t, SS for } \\
\text { he ratio o }\end{array}$ & $\begin{array}{l}\text { phosis, L } \\
\text { ral slope } \\
\text { laximal I }\end{array}$ & $\begin{array}{l}\text { ax for } \\
\text { /PI fo } \\
\text { oar lor }\end{array}$ & $\begin{array}{l}\text { aximal } \\
\text { e ratio } \\
\text { is divi }\end{array}$ & $\begin{array}{l}\text { nbar lc } \\
\text { pelvic } \\
\text { by pe }\end{array}$ & $\begin{array}{l}\text { osis, PI f } \\
\text { divided } \\
\text { inciden }\end{array}$ & $\begin{array}{l}\text { pelvic } \\
\text { pelvic }\end{array}$ \\
\hline
\end{tabular}

\section{Figures}




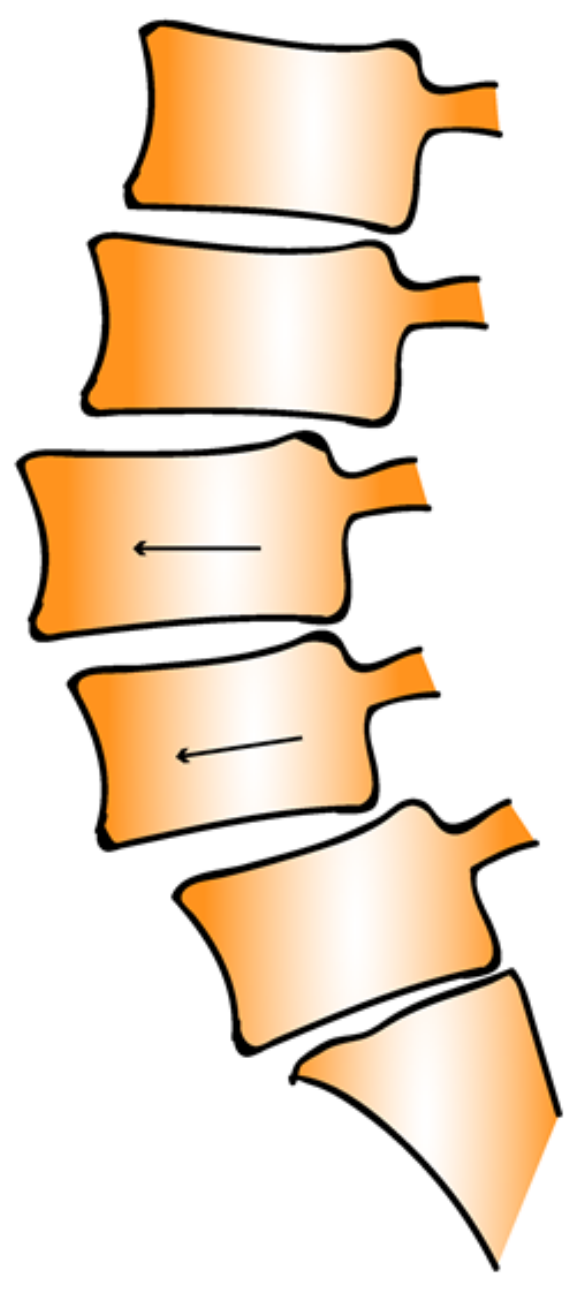

Anterior type

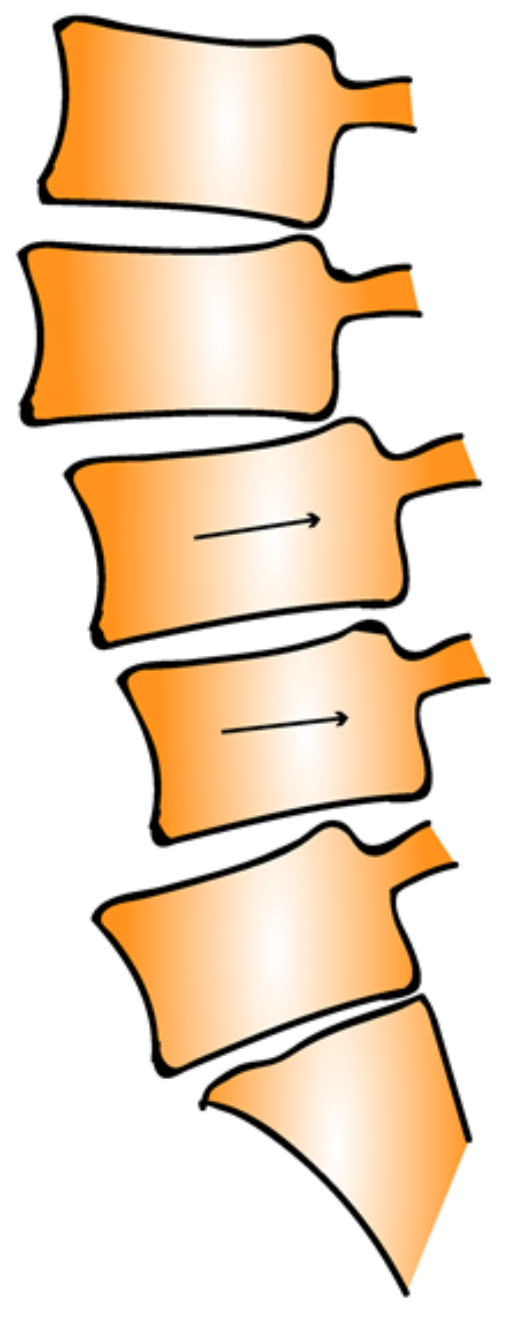

Posterior type

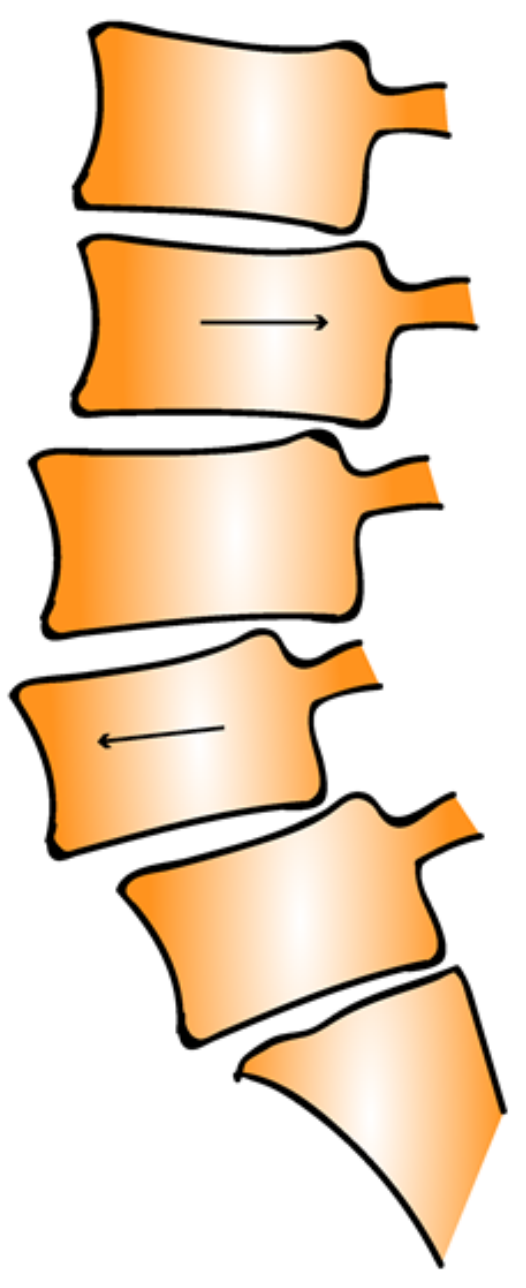

Combined type

Figure 1

Classification of the double-level DLS. Anterior type: anterolisthesis of two neighbouring vertebral bodies $\llbracket$ Posterior type: retrolisthesis of two neighbouring vertebral bodies (the incidence of this type is low and clinically rare); combined type: combination of anterolisthesis (usually seen in lower vertebral body) and anterolisthesis (upper vertebral body) 
a

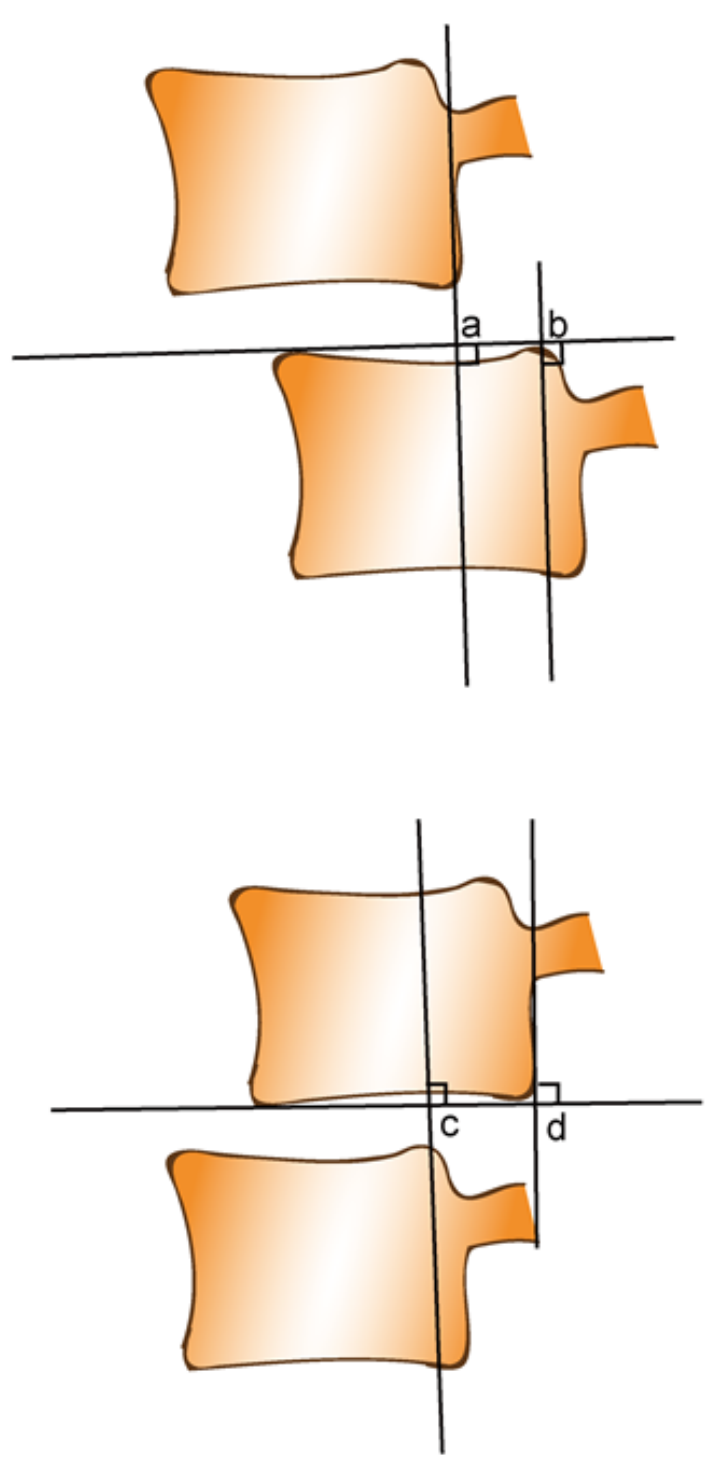

b

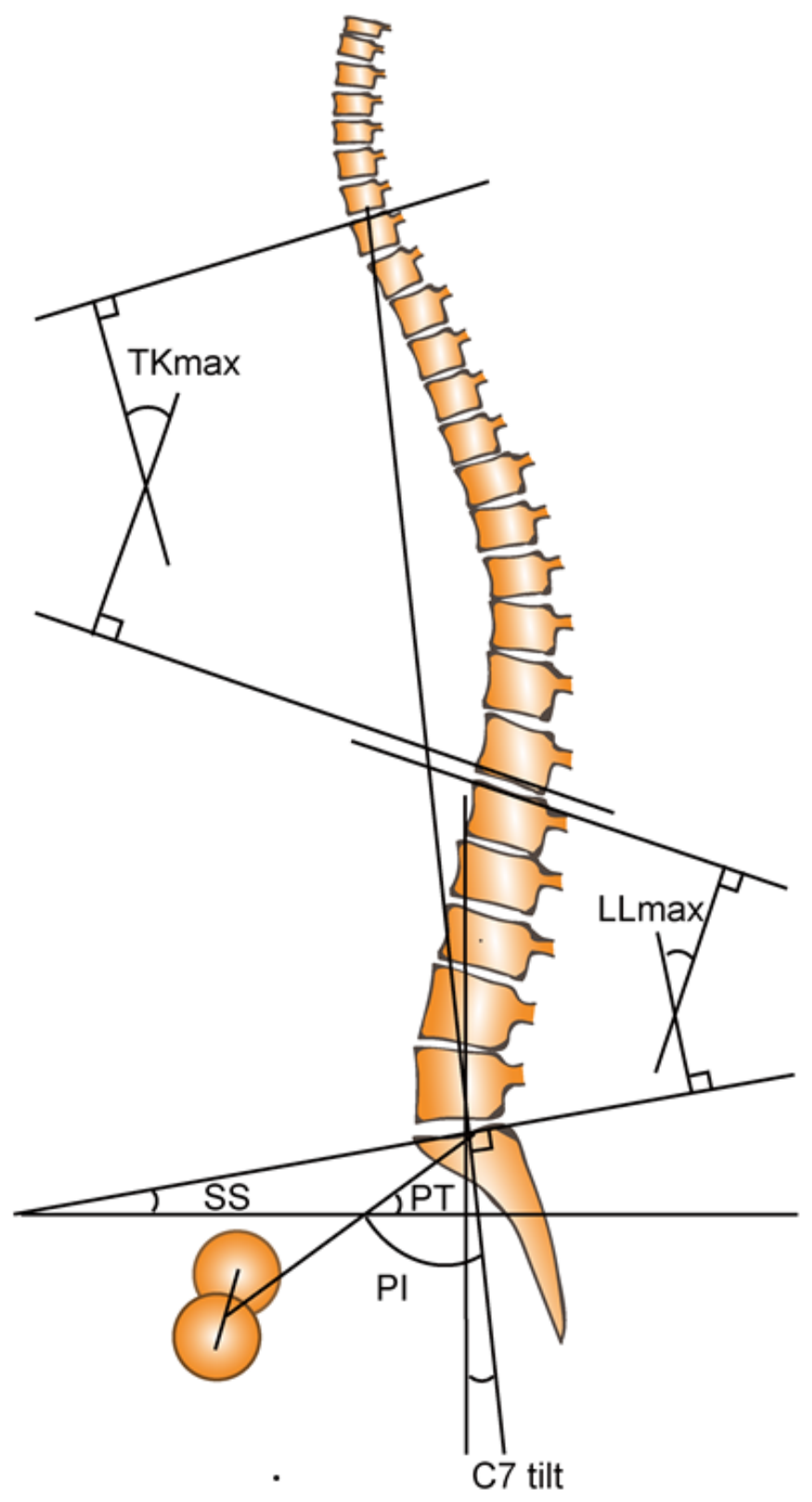

Figure 2

Measurement of the spondylolisthesis distance and sagittal spinopelvic parameters. A: Measurement of the vertebral body anterolisthesis (the line passing through the spondylolisthesis and the posterior edge of the inferior vertebral body intersects the tangent to the upper endplate of the inferior vertebral body at points $a$ and $b$, and measuring the distance between $a$ and $b$ ) and retrolisthesis (the line passing through the vertebral body of the spondylolisthesis and the posterior edge of the upper vertebral body perpendicular to the tangent line of the lower edge of the upper endplate at points $c$ and $d$, and measuring the distance between $\mathrm{C}$ and d) B: Measurement of the sagittal spinopelvic parameters: $\mathrm{C} 7 \mathrm{tilt}$, Maximal thoracic kyphosis (TKmax), Maximal lumbar lordosis (LLmax), Pelvic incidence (PI), Pelvic tilt (PT), Sacral slope (SS) 


\section{Supplementary Files}

This is a list of supplementary files associated with this preprint. Click to download.

- ConflictofInterestDisclosureForm.jpg 